\title{
Gestión de residuos en diálisis peritoneal: presente y futuro
}

\author{
M. Rosario Zugasti Laquidain* - M. Jesús Salsamendi Ballesteros* - Coro Melero Múgica* - M. Teresa \\ Rivas Osés* - Amaia Telleria Izaguirre* - Asier Vilas Gómez**
}

*DUE especialista en Urología-Nefrología

**Administrativo Servicio de Nefrología. Hospital Donostia. San Sebastián

\section{Resumen}

El impacto medioambiental de los residuos es motivo de preocupación en nuestra comunidad. Hemos estudiado a 26 pacientes en tratamiento con Diálisis Peritoneal con el fin de conocer el comportamiento de éstos con respecto a los residuos generados en la realización de su tratamiento y su interés en colaborar en proyectos de mejora en este campo en el futuro, así como posibles estrategias para conseguirlo.

Se elaboró un cuestionario para obtener la información necesaria y se cumplimentó mediante entrevista personal, voluntaria y confidencial. EI comportamiento de los pacientes con respecto a los residuos ha sido calificado de correcto a tenor de los resultados obtenidos, con la excepción de la recogida de los elementos punzantes (agujas), ya que el $46 \%$ (12) de los pacientes entrevistados los depositaba encapuchados en la bolsa de basura exclusiva para la recogida de residuos del tratamiento de Diálisis Peritoneal. La mayoría de los pacientes están dispuestos a colaborar en proyectos responsables con el tratamiento de residuos.

\section{PALABRAS CLAVE:}

- GESTIÓN DE RESIDUOS

- DIÁLISIS PERITONEAL

- ENVASES

- RESIDUOS SANITARIOS

Correspondencia:

M. Rosario Zugasti Laquidain

Servicio de Nefrología - Hospital Donostia

Paseo Dr. Begiristain s/n.

20014 Donostia (Gipuzkoa)

HDO.HEMODIALISIS@osakidetza.net
Waste management in peritoneal dialysis: present and future

\section{Abstract}

The environmental impact of waste is a cause for concern in our community. We have studied 26 patients undergoing Peritoneal Dialysis in order to find out their behaviour with regard to the waste generated in carrying out their treatment and their interest in collaborating in projects for improving this area in the future, as well as possible strategies to achieve this.

A questionnaire was drawn up to obtain the necessary information and was completed through a voluntary and confidential personal interview. The patients' behaviour in relation to waste was considered correct on the basis of the results obtained, except for the collection of sharp object (needles), as $46 \%$ (12) of the patients interviewed placed the needles properly covered in the exclusive bag for the collection of waste from Peritoneal Dialysis treatment. Most of the patients are willing to collaborate in waste treatment projects.

\section{KEY WORDS:}
- WASTE MANAGEMENT
- PERITONEAL DIALYSIS
- PACKAGING
- HEALTH WASTE

\section{Introducción}

Desde hace unos años, la preocupación por el impacto medioambiental de los residuos ha ido creciendo en nuestra comunidad gracias a las campañas de concienciación dirigidas por organismos públicos y privados. 
Desde un profundo respeto al paciente renal y a las personas que trabajamos para que reciban un tratamiento de calidad, y siendo conscientes de lo controvertido y complejo que resulta el tema, hemos querido asomarnos al mismo con nuestros deseos de aportar un punto de reflexión basado en nuestra experiencia y la de los profesionales que nos han asesorado. Recordemos que en España aproximadamente 2.200 pacientes que sufren Insuficiencia Renal Crónica Terminal (IRCT) están siendo tratados con Diálisis Peritoneal (DP) en la actualidad, por lo que la cantidad de residuos (toda materia que queda y se desecha después de realizar el tratamiento de DP) que generan es muy importante.

\section{Objetivo}

Conocer el comportamiento de los pacientes a la hora de la recogida y depósito de residuos generados en la aplicación de su tratamiento con DP, así como su disposición ante posibles estrategias que conduzcan al tratamiento responsable de los residuos.

\section{Material y Métodos}

Hemos encuestado a un grupo de 26 pacientes, elegidos de forma aleatoria y con edades comprendidas entre 24 y 80 años.

Para documentarnos sobre el tema utilizamos tres vías de información: a) solicitamos documentación escrita sobre la naturaleza de los materiales a dos de las empresas fabricantes recibiendo una amplia información por parte de ambas; b) mantuvimos una reunión conjunta con algunos de los responsables de los departamentos de Medio Ambiente y Salud Pública de nuestra comunidad y c) realizamos una serie de consultas al Departamento de Medicina Preventiva del Hospital.

Se diseñó un cuestionario para recoger la información necesaria y se cumplimentó mediante una entrevista personal, voluntaria y confidencial, en el propio hospital a 25 pacientes; uno lo rellenó en su domicilio debido a su limitación auditiva. Se empleó el método de la entrevista personal por considerar que, teniendo en cuenta el tema a tratar, pudiera ser necesario aclarar determinados conceptos y matizar preguntas y respuestas, de modo que los resultados pudieran ser tratados con mayor rigurosidad.
La elaboración del cuestionario se basó en el proceso de generación de residuos representado en la figura 1 . EI cuestionario se componía de seis apartados (anexol):

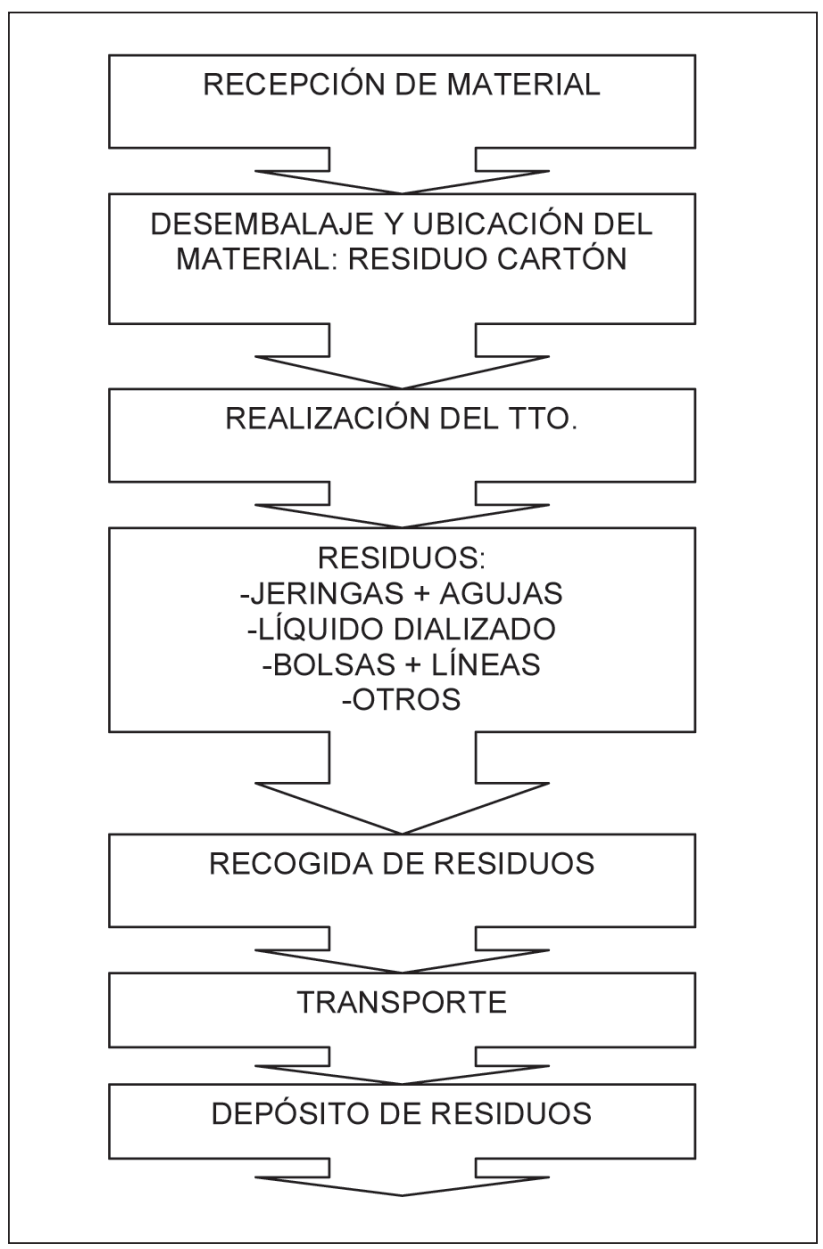

Figura 1. Proceso de generación de residuos.

a) Tipo de residuos y forma de recogida. Nos hemos centrado fundamentalmente en las jeringas+agujas, las bolsas con sus correspondientes líneas (residuos sólidos), y el líquido dializado (residuo líquido)

b) Almacenamiento

c) Persona(s) ocupada(s) de recoger y depositar los residuos

d) Evaluación de la dificultad

e) Disposición por parte del paciente o familiar hacia hipotéticos cambios en la ejecución del tratamiento y/o depósito de residuos.

f) Problemas y dificultades percibidas durante la entrevista (a rellenar por el entrevistador). 
[ M. R. Zugasti Laquidain, et al ]

Gestión de residuos en diálisis peritoneal: presente y futuro

\section{ANEXO I}

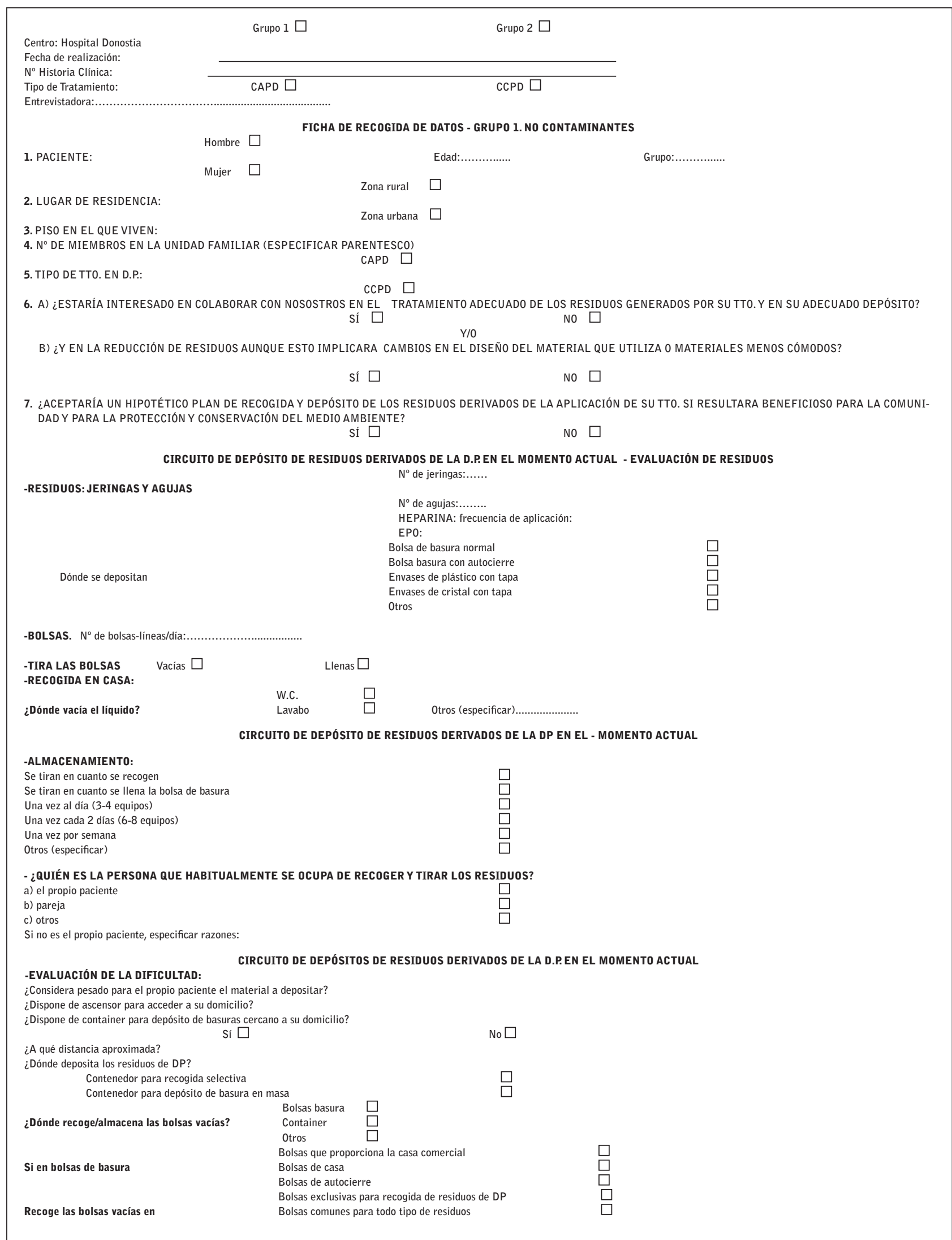




\section{Resultados}

De los 26 pacientes entrevistados, 24 residen en medio urbano y 2 en medio rural. 15 pacientes ( 7 mujeres y 8 hombres) utilizaban cicladora automática y 11 pacientes ( 1 mujer y 10 hombres) se trataba con DP manual.

Respecto a las respuestas del cuestionario cabe destacar:

a) Con respecto al cartón del embalaje, 24 pacientes (92\%) lo pliegan y lo depositan en el contenedor para recogida selectiva correspondiente. Uno de los pacientes lo utiliza para obtener energía y otro paciente no contesta. Cinco pacientes manifiestan tener dificultad para plegar los embalajes por su rigidez y dureza.

b) Todos los pacientes utilizaban jeringas precargadas con aguja integrada para heparina y eritropoyetina. Un paciente se inyectaba insulina pero utilizaba el método del bolígrafo. Doce pacientes $(46 \%)$ las depositaban en bolsas de basura junto con el resto del material. También 12 pacientes las depositaban en un contenedor con cierre de tapa (cristal y plástico) y lo entregaban en su centro de salud (dos pacientes acudían al centro de salud para la administración de la eritropoyetina).

c) Todos los pacientes vaciaban por el WC tanto el líquido dializado como el excedente de líquido de diálisis.

d) Todos los pacientes, una vez finalizado el tratamiento, recogían los equipos usados de DP (bolsas y líneas) en la bolsa de basura con autocierre proporcionada por la empresa proveedora. El 96\% (5) de los pacientes utilizaban las bolsas proporcionadas por la empresa proveedora para uso exclusivo de residuos de DP. Un paciente la utilizaba para todo tipo de residuos. El $46 \%$ (12) de los pacientes depositaban los residuos en el contenedor municipal en cuanto los recogían. El 54\% (14) de los pacientes lo hacían cuando se Ilenaba la bolsa.

e) La persona que habitualmente se encargaba de recoger y depositar los residuos era: en el $73 \%$ (19) de los casos, el propio paciente y en el $27 \%$ (7) la pareja u otros componentes de la unidad familiar.

f) Cinco pacientes consideraron pesado el material a depositar. Ninguno de ellos padecía limitación física alguna, ni una edad que pudiera incidir en el transporte de los residuos. Veintiún pacientes consideraron que el material no era pesado, mientras que uno manifestó que era voluminoso el $73 \%$ (19) de los pacientes disponían de ascensor en su domicilio y siete no tenían.

g) Veinticinco pacientes disponían de contenedor para depósito de residuos cerca de su domicilio, tanto para la recogida selectiva como para el resto de residuos en un área inferior a 100 metros de distancia. Un paciente, al residir en el medio rural, manifestó que la distancia hasta el contenedor más cercano era de aproximadamente $1 \mathrm{~km}$.

h) Con respecto a los equipos usados: el $81 \%$ (21) de los pacientes los depositaban en el contenedor de basura en masa. Cuatro pacientes lo depositaban en el contenedor para recogida selectiva (plástico) y uno no contesta.

i) De los pacientes entrevistados cinco mostraron espontáneamente su preocupación al respecto del tratamiento adecuado de los residuos.

La disposición de los pacientes ante hipotéticos cambios en el futuro se reflejó en las siguientes respuestas al cuestionario:

1. El 96\% (25) de los pacientes manifestaron estar interesados en colaborar con nuestro equipo en el tratamiento adecuado de los residuos y uno respondió que no estaba interesado.

2. Diez y ocho pacientes manifestaron estar interesados en colaborar en proyectos para la reducción de residuos, aún en el supuesto de que esto implicara cambios en el diseño del material o que éste resultara más incómodo para la realización del tratamiento, tres manifestaron no estar interesados y cinco no quisieron pronunciarse al respecto. Éstos expresaron dudas y reservas, anteponiendo la prevalencia del confort en los materiales sobre el objetivo. 
3. Veinticuatro pacientes aceptarían un hipotético plan de tratamiento de residuos si éste resultara beneficioso para la comunidad y para la conservación del medio ambiente, uno respondió negativamente y otro, condicionó la respuesta a la comodidad del proceso.

\section{Discusión y Conclusiones}

Como parte responsable del tratamiento con DP, nos preguntamos si el tratamiento de los residuos era adecuado, o por el contrario, estábamos pasando por alto la posibilidad de que todo o parte del material desechado se pudiera reciclar para su aprovechamiento.

Hemos tomado como referentes la Normativa Europea y la del País Vasco relativa a residuos sanitarios y envases. Esta última normativa sería aplicable según el Real Decreto 76/2002 por el que se regulan las condiciones para la gestión de los residuos sanitarios en la Comunidad Autónoma del País Vasco. Si consideramos las bolsas de DP como residuos sanitarios, quedarían clasificadas en el grupo 1 , 0 residuos sanitarios sin riesgo o inespecíficos, asimilables a residuos municipales o urbanos. En definitiva, recibirían el mismo tratamiento que los residuos domésticos, incluyendo su depósito.

No sería aconsejable reciclar la parte del equipo que hubiese estado en contacto con el dializado por tratarse de residuos sanitarios en contacto con líquidos orgánicos y, obviamente, podría existir la posibilidad de encontrarse el paciente en el periodo ventana de una enfermedad infecciosa. La Normativa Europea (Directiva 2004/12/CE) relativa a envases y residuos de envases ha sido mas difícil de interpretar ya que la consideración o no como envase de las bolsas de DP ha sido controvertida. Por tanto, sólo se reciclan envases, y tomando como referencia la Directiva 2004/12/CE no tenemos base suficiente para aplicar con toda seguridad la categoría de envase a las bolsas de DP. Por lo tanto, en nuestra opinión, no es viable en la actualidad la alternativa del reciclado de estos residuos por la vía de la recogida selectiva municipal y las bolsas de DP, al no poder ser recicladas por esta vía deberían ser depositadas en el contenedor de basura en masa para su posterior valorización 0 eliminación. Como consecuencia, la estrategia a seguir sería la de la reducción de los residuos en la medida de lo posible y la implicación de las empresas fabricantes para conseguir materiales que minimicen el impacto ambiental.

Ante la propuesta del estudio, todos los pacientes aceptaron responder a las preguntas del cuestionario. Varios de los pacientes manifestaron dudas sobre la utilidad del estudio en el apartado de estrategias a adoptar en el futuro, ya que consideraban que los residuos derivados de la DP son secundarios en comparación con los graves problemas provocados por otro tipo de residuos. En varios de los entrevistados se percibió cierto temor al formular la pregunta que hacía referencia a los hipotéticos cambios en la comodidad del material o en su diseño, pues pensaron que quizás la respuesta podría perjudicarles en el futuro.

Nos ha sorprendido el dato de que casi la mitad de los pacientes no cumplían con el protocolo de recogida de materiales punzantes en el que se les había entrenado (recipientes cerrados con tapa a rosca). El comportamiento de los pacientes en cuanto al resto de los residuos ha sido bastante homogéneo y lo hemos calificado como correcto ya que todos los pacientes disponían de los elementos necesarios para una recogida adecuada, y los puntos de depósito (contenedor municipal) estaban muy próximos al domicilio.

En la actualidad teniendo en cuenta que no es viable la alternativa del reciclado tradicional para estos residuos deberíamos incluir en el entrenamiento a los pacientes la educación sobre hábitos responsables en la utilización de los recursos, habida cuenta su disposición mayoritariamente favorable.

Creemos firmemente que la relación calidad-seguridad de los recursos y la rentabilidad económica pueden ser compatibles con el respeto al medio ambiente. Animamos, por ello, a los fabricantes a que continúen investigando en materiales, formatos y sistemas de gestión para que en el futuro, al menos una parte de estos residuos, puedan ser reciclados. EI manejo de los residuos es responsabilidad de todos; por lo tanto deberemos trabajar todos juntos para encontrar el equilibrio entre un tratamiento de calidad y el respeto al medio ambiente. 
Para finalizar recordamos la importancia de cumplir estas premisas fundamentales:

Utiliza sólo lo que necesites. Recuerda que si cuidas el planeta, el planeta te cuidará a ti.

\section{Agradecimientos}

A D. Javier Ansorena Miner, D. José Ramón Sáez Domínguez, y Da. Itziar Zaldua Echave por ayudarnos a descubrir la cara desconocida de los residuos. A todos los pacientes que han colaborado en este estudio.

\section{Bibliografía}

1. Decreto 76/2002 del 26 de marzo, por el que se regulan las condiciones para la Gestión de los residuos sanitarios en la Comunidad Autónoma del País Vasco, capítulo I, artículos 1,2 y 3. (B0E n ${ }^{\circ}$ 75, del 22 de abril de 2002).

2. Directiva $2004 / 12 / C E$ del 11 de febrero de 2004, por la que se modifica la Directiva 94/62/CE relativa a los envases y residuos de envases.

3. Directiva 75/442/CEE relativa a los residuos (Catálogo Europeo de Residuos): letra A del artículo 1.

4. Lista Europea de Residuos. Anejo 2 de la Orden MAM/304/2002 del 8 de febrero (B0E n ${ }^{\circ} 43$ del 19 de febrero de 2002 y corrección de errores BOE $n^{\circ} 61$ del 12 de marzo de 2002).

5. Orden MAM/3624/2006 relativa a Envases y Residuos de envases. Páginas 41.813-41.815. Referencias 2006/20766. (BOE n 285 del 29 de noviembre de 2006). 